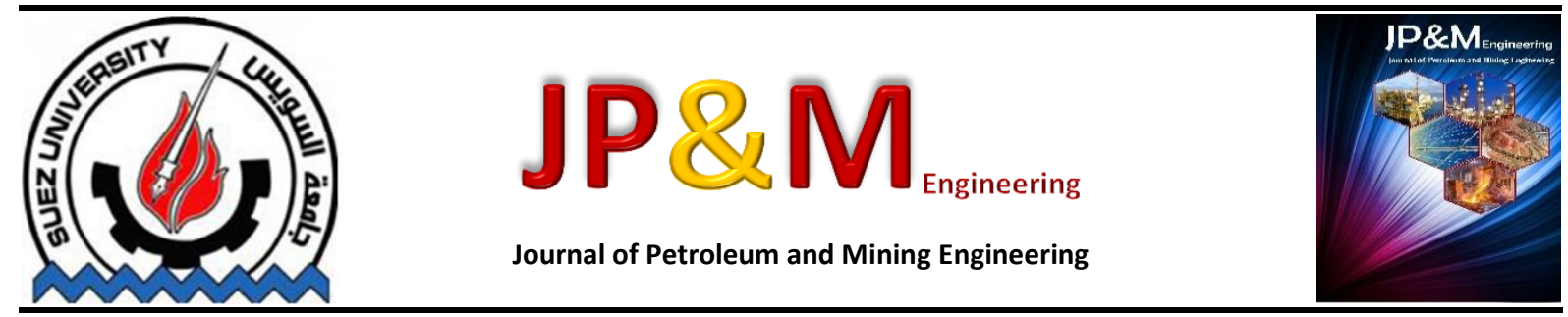

\title{
Evaluation of INS Performance in Mine Mapping
}

\author{
A. M. Zayed, ${ }^{*}$ M. A. Hamama ${ }^{b}$, H. E. Elhifnawy and A. M. Kamel ${ }^{\text {d }}$ \\ ${ }^{a, b}$ Faculty of Petroleum and Mining Engineering, Suez University, Egypt \\ 'Civil Engineering Department, Military Technical College \\ ${ }^{\mathrm{d}}$ Guidance Department, Military Technical College
}

\section{Keywords}

Mine; INS; IMU; Sensor errors

\begin{abstract}
This research paper discusses and evaluates the feasibility of using Inertial Navigation System (INS) in mine tracking, which is necessary for both surface and underground mining operations. INS provides position, velocity and attitude through direct measurements from inertial sensors (Accelerometers and Gyroscopes). INS suffers from time-dependent error growth which causes a drift in the object track, thus compromising the long-term accuracy of the system. In order to validate the INS algorithm, a toolbox developed under MATLAB environment was used to generate a reference and INS trajectories. Deep investigation and analysis of the affected error sources of INS has been conducted. The attained results indicated that the accuracy of INS depends upon the error sources, so it is important to integrate INS with other sensor output (as GPS) to minimize the effect of sensor drifts.
\end{abstract}

\section{Introduction}

Inertial navigation is a process whereby the measurements provided from the inertial sensors (Gyroscopes and Accelerometers) to obtain the position of a moving object in which they are installed. So, Inertial Navigation System (INS) is a self-contained navigation system within the vehicle that is undependable on the transmission of signals from the vehicle or reception from external sources. Inertial Navigation System (INS) typically contains three orthogonal gyroscopes for measuring angular velocity and three orthogonal accelerometers for measuring linear acceleration [1], [2].

Inertial navigation is used in a wide range of applications including the navigation of aircraft, tactical and strategic missiles, spacecraft, submarines and ships. Recent advances in the construction of Micro-Electro Mechanical Systems (MEMS) devices have made it possible to manufacture small and light inertial navigation systems. These advances have widened the range of possible applications to include areas such as human and animal motion tracking [3], [1].

\section{Mechanization Equations}

Inertial Navigation System (INS) consists of three accelerometers and three gyroscopes mounted on an orthogonal triad. The accelerometers measure the specific force defined in the inertial frame as mentioned in equation (1) [2], [4]:

$$
f=a-g
$$

Where $f$ is the specific force, $a$ is the acceleration and $g$ is the gravitational acceleration.

The velocity of the moving object can be obtained by first integration and the result added to the initial velocity. The position of the moving object is obtained by integrating the equation twice then adds the result to the initial position [2].

The mechanization equations are a set of equations used to obtain useful navigation information from the INS outputs (specific force and angular rate). In this paper, mechanization will be implemented in Easting, Northing and Upper directions (ENU) frame as a navigation frame.

Equation (2) shows the mathematical model for the equations of motion of an object [3], [5], [6]:

$$
\left(\begin{array}{l}
i^{n} \\
v^{n} \\
R_{b}^{n}
\end{array}\right)=\left[\begin{array}{c}
D^{-1} v^{n} \\
R_{b}^{n} f^{b}-\left(2 \Omega_{i e}^{n}+\Omega_{e n}^{n}\right) v^{n}+g^{n} \\
R_{b}^{n}\left(\Omega_{i b}^{b}-\Omega_{i n}^{b}\right)
\end{array}\right]
$$

The first part of equation (2) represents the relationship between the geographic coordinates and the velocity components in the ENU frame as shown in equation (3)[6], [7]. 


$$
\begin{aligned}
& r^{n}=\left[\begin{array}{l}
\varphi \\
\lambda \\
h
\end{array}\right]=D^{-1} v^{n}= \\
& {\left[\begin{array}{ccc}
0 & \frac{1}{M+h} & 0 \\
\frac{1}{(N+h) \cos \varphi} & 0 & 0 \\
0 & 0 & 0
\end{array}\right]\left[\begin{array}{l}
v^{E} \\
v^{N} \\
v^{U}
\end{array}\right]}
\end{aligned}
$$

The second part of same equation represents the time derivative of the velocity where $\Omega_{\mathrm{ie}}^{\mathrm{n}}$ and $\Omega_{\mathrm{en}}^{\mathrm{n}}$ the orientation parameters that are derived as the skew symmetric matrices of the vectors in equations (4) and (5). $\mathrm{f}^{\mathrm{b}}$ is the specific force vector measured by the accelerometer in the body frame (b-frame) [4], [7].

$$
\begin{aligned}
& \omega_{i e}^{n}=R_{e}^{n} \omega_{i e}^{e}= \\
& {\left[\begin{array}{ccc}
-\sin \lambda & \cos \lambda & 0 \\
-\sin \varphi \cos \lambda & -\sin \varphi \sin \lambda & \cos \varphi \\
\cos \varphi \cos \lambda & \cos \varphi \sin \lambda & \sin \varphi
\end{array}\right]\left[\begin{array}{l}
0 \\
0 \\
\omega_{e}
\end{array}\right]=} \\
& {\left[\begin{array}{c}
0 \\
\omega_{e} \cos \varphi \\
\omega_{e} \sin \varphi
\end{array}\right]}
\end{aligned}
$$

$$
g^{n}=\left[\begin{array}{r}
0 \\
0 \\
-g
\end{array}\right]
$$

The third part of mechanization equation represents the attitude mechanization equation and expresses the dynamics of the attitude of the b-frame with respect to $n$-frame where $\Omega_{\text {in }}^{b}$ is the skew symmetric matrix of the vectors in equation (7) and $\omega_{\mathrm{ib}}^{\mathrm{b}}$ is a vector of angular rates measured by the gyros [4], [8].

$$
\omega_{i n}^{b}=R_{b}^{n}\left[\begin{array}{c}
\frac{-v^{N}}{M+h} \\
\frac{v^{E}}{N+h}+\omega_{e} \cos \varphi \\
\frac{v^{E} \tan \varphi}{N+h} \omega_{e} \sin \varphi
\end{array}\right]
$$

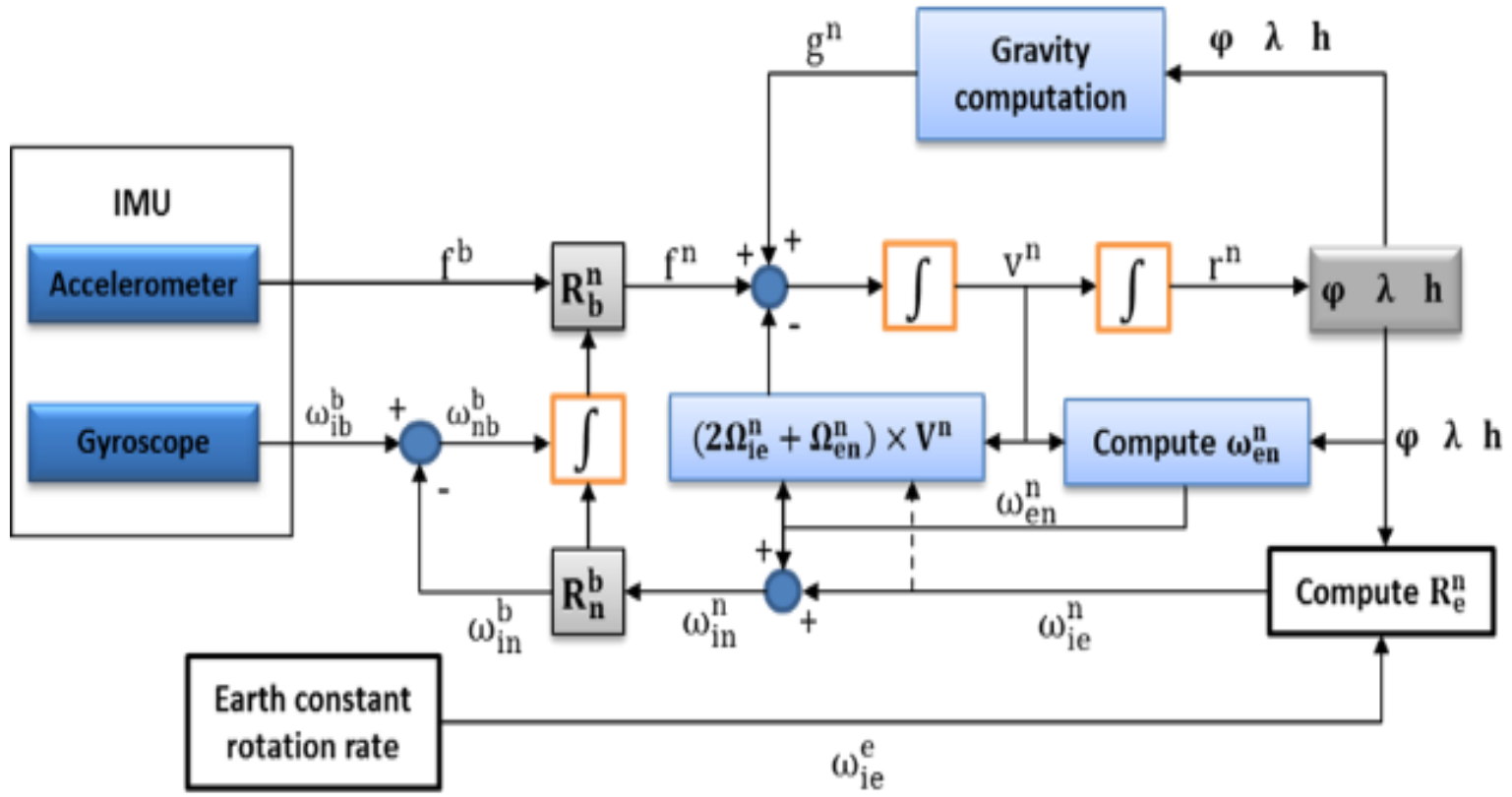

Figure 1 The INS navigation frame mechanization [3].

$$
\omega_{e}^{n}=\left[\begin{array}{l}
-\varphi \\
\lambda \cos \varphi \\
\lambda \sin \varphi
\end{array}\right]=\left[\begin{array}{l}
\frac{-v^{N}}{M+h} \\
\frac{v^{E}}{N+h} \\
\frac{v^{E} \tan \varphi}{N+h}
\end{array}\right]
$$

\section{INERTIAL}

\section{INERTIAL SENSOR ERRORS}

Measurement from both accelerometers and gyroscopes are subject to errors which limit the accuracy with measured observations. Sensor errors are represented as bias, scale factor, noise, repeatability and stability. These errors have to be determined with good understanding of their effects to be able to evaluate the performance of inertial sensors [7], [9]. 
Bias is the output that has no correlation with the input. Sensor bias is deterministic in nature and it is normally expressed in $(\mathrm{m} / \mathrm{s} 2$ or $\mathrm{mg}$ ) for accelerometers and [deg/hr or rad/s] for gyroscopes. Figure (1) shows a schematic diagram that summarizes the mechanization equations starting from inertial sensor measurements and end up with object position, velocity and attitudes at any time [3].

Scale factor is the ratio between the output signal of the sensor and the physical quantity being measured which is expressed in [ppm] (parts per million) for both accelerometers and gyroscopes. Both bias and scale factor can be determined by calibration. Noise exists as additional signals that can be resulted from the sensor itself or other electronic equipment that interferes with the measured output signals. Noise is in general non-systematic and therefore cannot be removed from the data using deterministic models but it can only be modeled by stochastic means [5].

Accelerometer and Gyroscope measurement models are represented in equations ( 8 and 9) [4]

$I_{f}=f+b_{f}+S_{1} f+S_{2} f^{2}+N f+\delta g+\varepsilon(f)$

Where:

$I_{f}$ is the accelerometer measurement $\left(\mathrm{m} / \mathrm{sec}^{2}\right)$.

$\mathrm{f}$ is the true specific force (Observable) $\left(\mathrm{m} / \mathrm{sec}^{2}\right)$.

$b_{f}$ the accelerometer instrument bias $\left(\mathrm{m} / \mathrm{sec}^{2}\right)$.

$\mathrm{S}_{1}$ a matrix of the linear scale factor error.

$\mathrm{S}_{2}$ a matrix of the non-linear scale factor error.

$\mathrm{N}$ a matrix representing axes non-orthogonality.

$\delta g$ the anomalous gravity vector (deviation from the

theoretical gravity value) ( $\left.\mathrm{m} / \mathrm{sec}^{2}\right)$.

$\varepsilon(\mathrm{f})$ is a vector representing the gyro sensor noise $\left(\mathrm{m} / \mathrm{sec}^{2}\right)$.

$I_{\omega}=\omega+b_{\omega}+S \omega+N \omega+\varepsilon(\omega)$

Where

$\mathrm{I}_{\omega}$ the gyroscope measurement (deg/hr)

$\omega$ the true angular velocity (The theoretically desired measurement) (deg/hr).

$\mathrm{b}_{\omega}$ the gyroscope bias (deg/hr).

$\mathrm{S}$ a matrix representing the gyroscope scale factor.

$\mathrm{N}$ is a matrix representing non-orthogonality of the gyros triad.

$\varepsilon(\omega)$ is a vector representing the gyro sensor noise (deg/hr).

\section{SIMULATION RESULTS}

In order to evaluate the accuracy of INS in mine mapping, LN-200 IMU was chosen as a used INS system which has the specifications illustrated in table 1. Reference trajectory was generated using a GPSoft toolbox under MATLAB environment. The reference trajectory will be tested under effects of sensor errors. Accelerometer bias, gyro drift, sensors scale factor and gyro noise were taken as case study and their effects on the derived INS trajectory will be derived.
Table 1 specifications of the Accelerometer and Gyro

\begin{tabular}{|l|l|l|}
\hline & Accelerometer & Gyro \\
\hline $\begin{array}{l}\text { Bias } \\
\text { Repeatability }\end{array}$ & $300 \mu \mathrm{g}$ & $0.5^{\circ} / \mathrm{hr}$ \\
\hline $\begin{array}{l}\text { Scale Factor } \\
\text { Accuracy }\end{array}$ & $100 \mathrm{ppm}$ & $100 \mathrm{ppm}$ \\
\hline $\begin{array}{l}\text { Random Walk } \\
\text { (max) }\end{array}$ & -------- & $\begin{array}{l}0.05^{\circ} / \sqrt{\mathrm{h}} \mathrm{P} \\
\text { Power Spectral } \\
\text { Density (PSD) } \\
\text { level }\end{array}$ \\
\hline
\end{tabular}

\section{Accelerometer bias effect}

In order to study the effect of the accelerometer bias on the computed trajectory, $300 \mu \mathrm{g}$.accelerometer bias is used. Figure (2) shows the reference and the derived INS trajectories after adding accelerometer bias. Accelerometer bias causes improper measurement of the accelerometers which in turn, results in improper computation in velocity and position as shown in figure (3).

\section{Gyroscope drift effect}

As in accelerometer bias effect, gyro drift has been selected as $0.50 / \mathrm{hr}$. Figure (4) shows the difference between the reference and the derived INS trajectories with gyro drift and figure (5) shows errors in horizontal and vertical position. Gyroscope drift of $0.5 \mathrm{o} / \mathrm{hr}$ results in RMSE in horizontal position of about $26.53 \mathrm{~m}$ and RMSE in vertical position of less than 8 $\mathrm{cm}$.

\section{Accelerometer scale factor error effect}

Accelerometer scale factor is selected as 100 ppm. There is a small impact of scale factor error on the derived INS trajectory. Scale factor error of 100 ppm results in RMSE in horizontal position of $1.49 \mathrm{~m}$ and in vertical position of $11 \mathrm{~cm}$.

\section{Gyro scale factor error effect}

As in accelerometer scale factor effect, gyro scale factor is chosen as $100 \mathrm{ppm}$ to illustrate its impact of the INS trajectory. The RMSE in horizontal position is less than $85 \mathrm{~cm}$

\section{Gyro white noise effect}

Gyro noise (Random walk) of used INS is $0.050 / \sqrt{\mathrm{hr}}$. The effect of this error on the performance of the navigation system is illustrated as RMSE in horizontal position which is less than $18 \mathrm{~m}$ while in vertical position is about $8 \mathrm{~cm}$.

\section{The effect of the all errors}

Finally, the performance INS is tested under the effects of all errors together. Figure (6) illustrates the difference between the reference and the INS trajectories with all errors. It is clear that the deviation of INS trajectory from the reference one is greater than the deviation resulting from any individual error. 


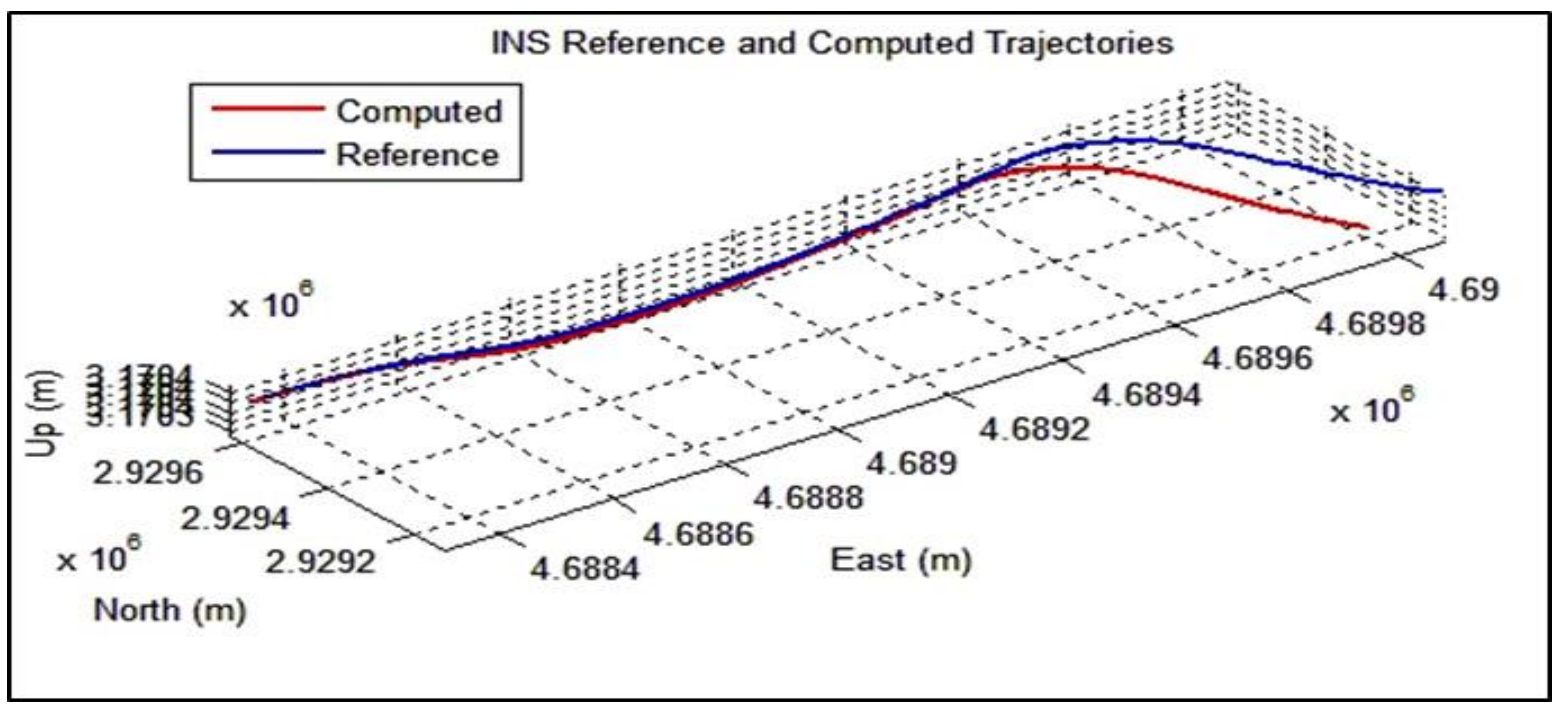

Figure 2 Reference and computed trajectories with $300 \mu \mathrm{g}$ accelerometer bias.

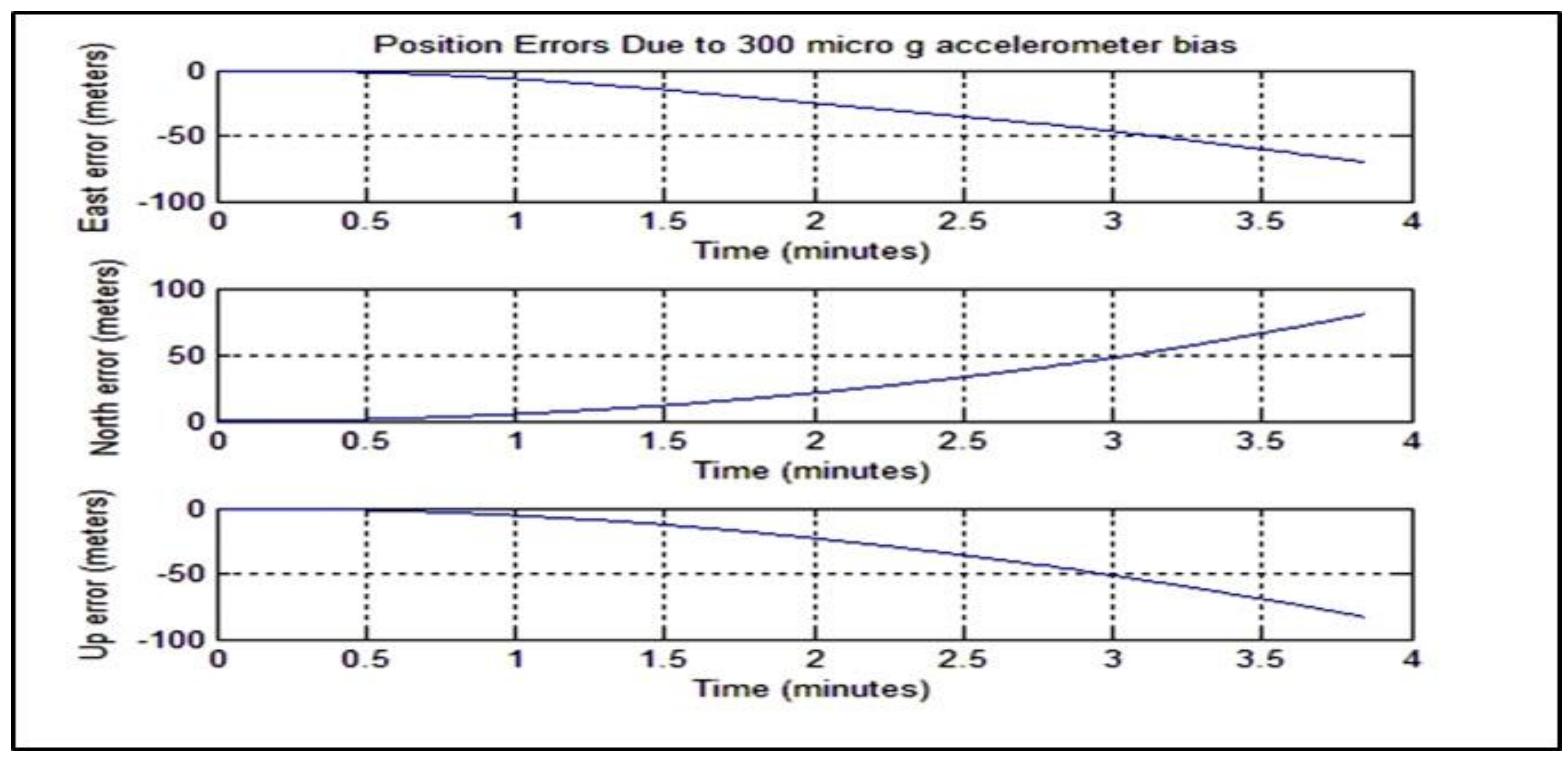

Figure 3 Horizontal and vertical position error due to accelerometer bias.

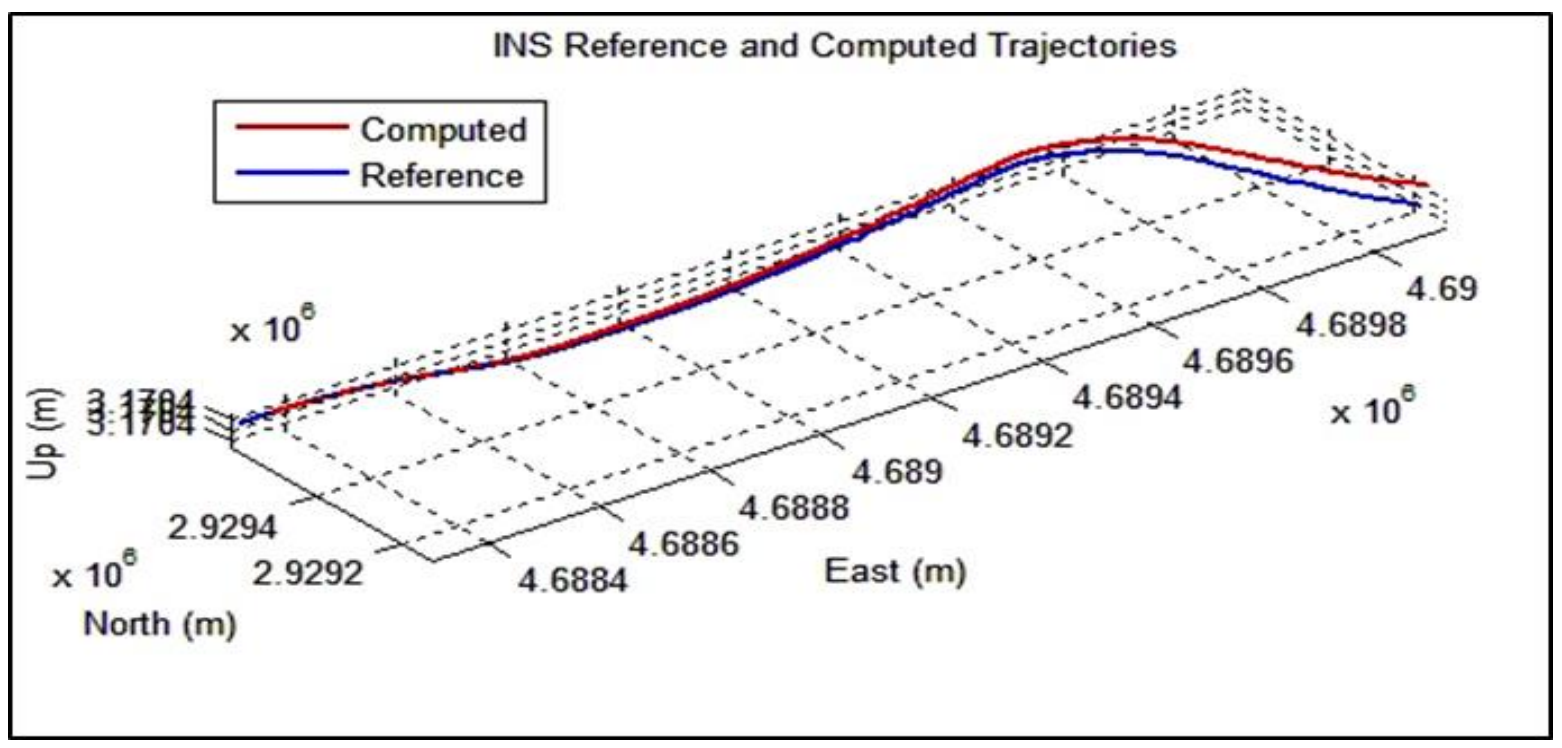

Figure 4 Reference and computed trajectories with $0.50 / \mathrm{hr}$ accelerometer bias. 

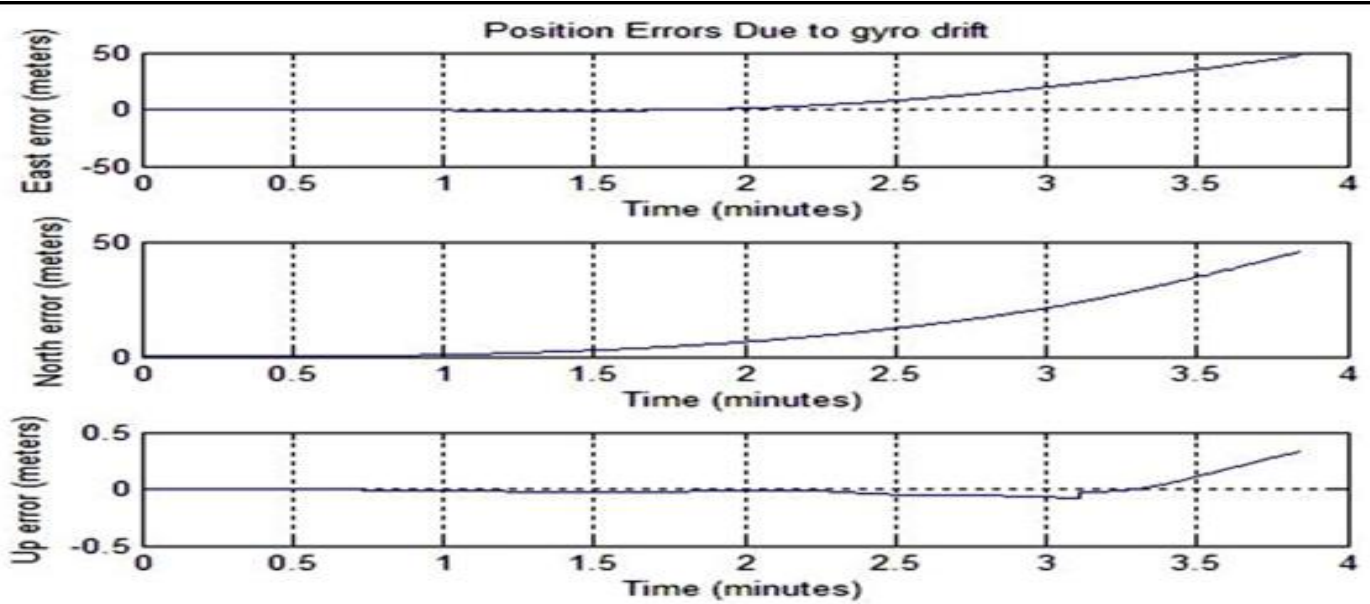

Figure 5 Horizontal and vertical position errors due to gyro drift.

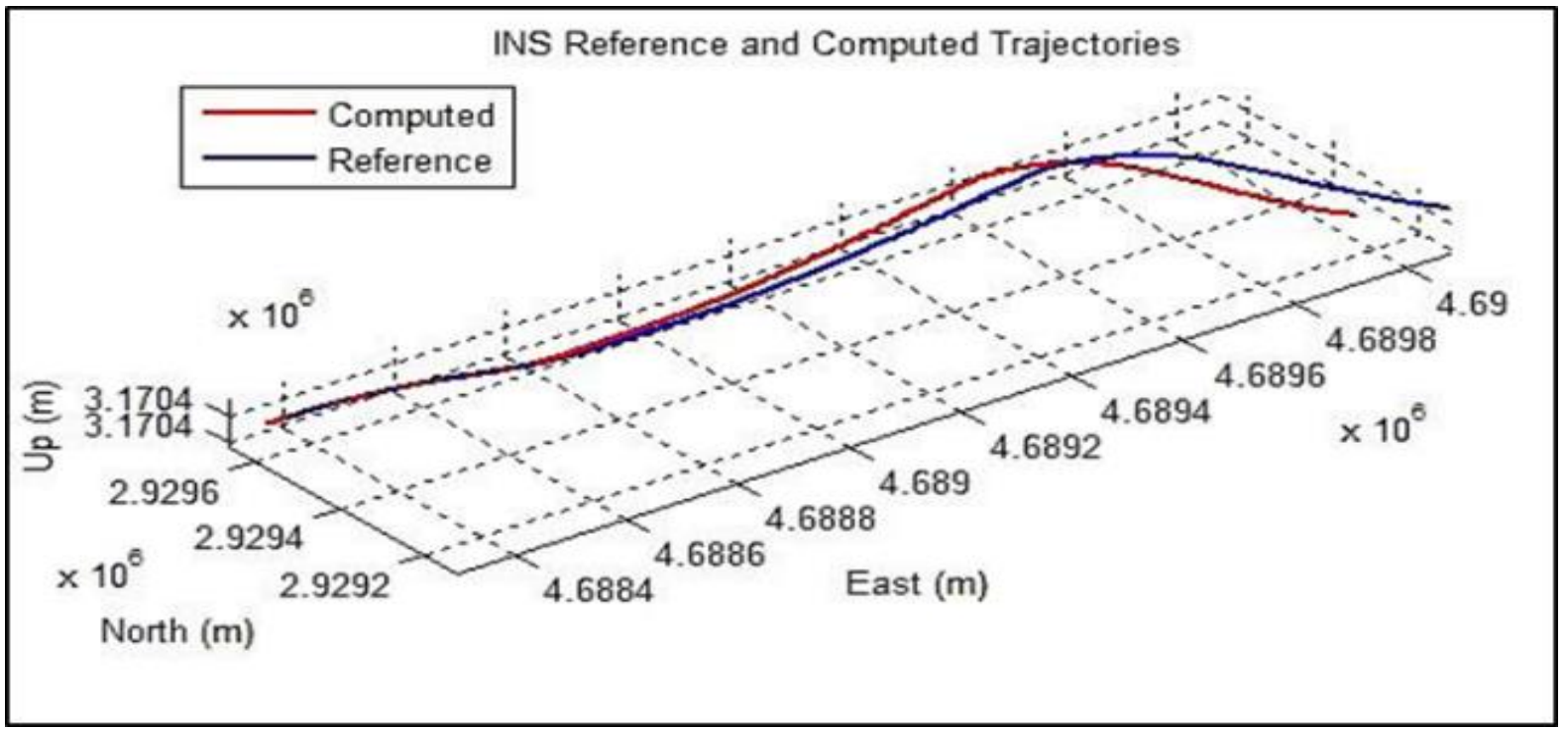

Figure 6 Reference and computed trajectories with all errors

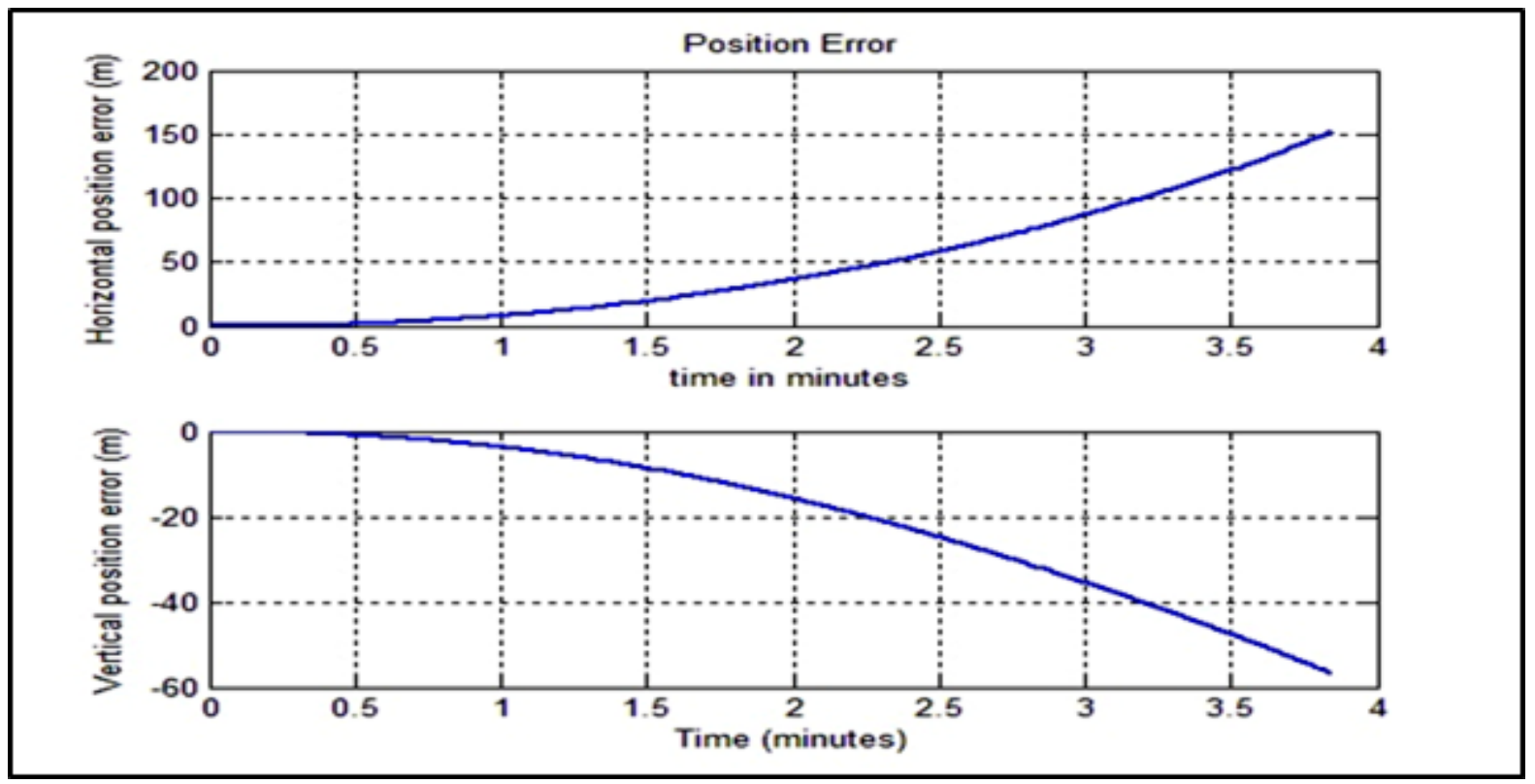

Figure 7 Horizontal and vertical position error due to all errors 


\section{Conclusions}

INS is used to get rich and dense information for moving object, position, velocity and attitude. Sensor error sources play a great role in INS efficiency in case of individual effect or accumulated due to effects of all sensor error at the same time. RMSE due to all errors together in horizontal position equal $67.3 \mathrm{~m}$ and in vertical position $26.8 \mathrm{~m}$. So, other positioning sensor has to be used with INS to reduce the sensor errors effects and to improve its outputs. GPS is the most positioning sensor.

suitable to be integrated with INS to minimize error sensor effects because its derived position is not contaminated with error sources like INS and it is more accurate. GPS is suitable for INS/GPS integration in open area mining processes but it is not suitable for underground use since there is no GPS signal. In underground fields, odometer or fixed ground control points (GCPs) are suitable for integration process to minimize sensor errors effects in order to be used in mine mapping.

\section{REFERENCES}

[1] J.Woodman, O. "An introduction to inertial navigation", 2007, Technical reports published by the University of Cambridge.

[2] Titterton, D., and Weston, J.L "Strapdown Inertial Navigation Technology" 2007, American Institute of Aeronautics and Astronomy (AIAA).

[3] Schultz, C.E. "INS and GPS integration", 2006, Informatics and Mathematical Modeling, Technical University of Denmark.

[4] El-Sheimy "Inertial techniques and INS/DGPS Integration", 2004, Department of Geomatics Engineering, University of Calgary, Canada.

[5] El-Sheimy "MEMS-Based Integrated Navigation" first edition, 2010, Artech House.

[6] Eun-Hwan Shin "Accuracy Improvement of Low Cost INS/GPS for Land Applications", 2001, Department of Geomatics Engineering, University of Calgary, Canada.

[7] A. Noureldin, T. Karamat and J.Georgy "Fundamentals of Inertial Navigation, Satellite-based Positioning and their Integration", 2013, Springer

[8] Godha, S. "Performance Evaluation of Low Cost MEMSBased IMU Integrated With GPS for Land Vehicle Navigation Application", 2006, Department of Geomatics Engineering, University of Calgary, Canada.

[9] Xiaoying Kong "Inertial Navigation System Algorithms for Low Cost IMU", 2000, Department of Mechanical and Mechatronic Engineering, University of Sydney 